\title{
Treatment Coverage Estimation for Mass Drug Administration for Malaria with Dihydroartemisinin-Piperaquine in Southern Province, Zambia
}

\author{
Timothy P. Finn, ${ }^{1}$ Joshua O. Yukich, ${ }^{1}$ Adam Bennett, ${ }^{2}$ Travis R. Porter, ${ }^{1}$ Christopher Lungu, ${ }^{3}$ Busiku Hamainza, ${ }^{4}$ \\ Elizabeth Chizema Kawesha, ${ }^{4}$ Ruben O. Conner, ${ }^{5}$ Kafula Silumbe, ${ }^{3}$ Richard W. Steketee, ${ }^{5}$ John M. Miller, ${ }^{3}$ Joseph Keating, ${ }^{1}$ \\ and Thomas P. Eisele ${ }^{1 *}$ \\ ${ }^{1}$ Department of Tropical Medicine, Center for Applied Malaria Research and Evaluation, Tulane University School of Public Health and Tropical \\ Medicine, New Orleans, Louisiana; ${ }^{2}$ Malaria Elimination Initiative, Global Health Group, University of California San Francisco, San Francisco, \\ California; ${ }^{3}$ PATH Malaria Control and Elimination Partnership in Africa (MACEPA), Lusaka, Zambia; ${ }^{4}$ National Malaria Elimination Centre, Zambia \\ Ministry of Health, Chainama Hospital, Lusaka, Zambia; ${ }^{5}$ PATH MACEPA, Seattle, Washington
}

\begin{abstract}
Mass drug administration (MDA) is currently being considered as an intervention in low-transmission areas to complement existing malaria control and elimination efforts. The effectiveness of any MDA strategy is dependent on achieving high epidemiologic coverage and participant adherence rates. A community-randomized controlled trial was conducted from November 2014 to March 2016 to evaluate the impact of four rounds of MDA or focal MDA (fMDA)where treatment was given to all eligible household members if anyone in the household had a positive malaria rapid diagnostic test—on malaria outcomes in Southern Province, Zambia (population approximately 300,000). This study examined epidemiologic coverage and program reach using capture-recapture and satellite enumeration methods to estimate the degree to which the trial reached targeted individuals. Overall, it was found that the percentage of households visited by campaign teams ranged from $62.9 \%(95 \% \mathrm{Cl}$ : $60.0-65.8)$ to a high of $77.4 \%$ (95\% Cl: $73.8-81.0)$ across four rounds of treatment. When the maximum number of visited households across all campaign rounds was used as the numerator, program reach for at least one visit would have been $86.4 \%$ (95\% Cl: 80.8-92.0) in MDA and 83.5\% (95\% Cl: 78.0-89.1) in fMDA trial arms. As per the protocol, the trial provided dihydroartemisinin-piperaquine treatment to an average of $58.8 \%$ and $13.3 \%$ of the estimated population based on capture-recapture in MDA and FMDA, respectively, across the four rounds.
\end{abstract}

\section{INTRODUCTION}

In coordination with the National Malaria Control Centre (NMCC) of Zambia, a community-randomized controlled trial was conducted in Southern Province to evaluate the impact of four rounds of mass drug administration (MDA) or focal MDA (fMDA), where treatment was given to all eligible household members if anyone in the house had a positive malaria rapid diagnostic test (RDT). ${ }^{1}$ Mass drug administration is conducted community wide, whereas FMDA is conducted at the household level. A key factor driving the effectiveness of MDA for interrupting malaria transmission is population coverage. ${ }^{2-6}$ Programs face challenges in reporting MDA coverage because of the difficulty in knowing the population denominator in areas where accurate household lists do not exist and official census data can be outdated or incongruent with program implementation boundaries. Accurately determining the proportion of individuals who were reached and who took the treatment course is critical for the evaluation of MDA; relying on programmatic records of drug distributors alone for who was enumerated and took treatment may provide an incomplete picture of how many target houses were not visited. ${ }^{7}$

Many MDA programs use after treatment coverage surveys (TCS) to validate reported MDA coverage. ${ }^{8-10}$ These surveys primarily use a probability two-stage cluster sampling method to estimate the treatment coverage by administering a timely and concise questionnaire to households. ${ }^{11}$ To estimate MDA coverage, surveys collect information to estimate both the numerator-the people reporting being treated or visited by

\footnotetext{
* Address correspondence to Thomas P. Eisele, Department of Tropical Medicine, Center for Applied Malaria Research and Evaluation, Tulane University School of Public Health and Tropical Medicine, 1440 Canal St., Suite 2200, New Orleans, LA 70112. E-mail: teisele@tulane.edu
}

the MDA campaign —and the denominator-the total people who should have been treated or visited.

The terminology for reporting MDA coverage is heterogeneous and markedly inconsistent in the literature and limits comparison across studies. ${ }^{12,13}$ Given the limited reporting of malaria MDA experiences at scale in the last 20 years, the evidence base for evaluating MDA coverage and measurement methodologies is challenging. ${ }^{14,15}$ To date, there have been no published studies assessing methods for estimating coverage for malaria MDA as has been performed for neglected tropical diseases. ${ }^{10,16-18}$

Capture-recapture techniques provide an alternative method to TCS for measuring MDA coverage. To our knowledge, capture-recapture, methods that estimate a denominator by comparing the overlap of independently collected population lists for the same population, ${ }^{19}$ has not previously been used for estimating the denominator for an MDA program. Primarily used in ecology to estimate the size of animal populations where censuses are difficult to conduct, capture-recapture has been used to estimate human population size as far back as $1802 .^{20}$ In public health, capture-recapture methods have been applied to assess the completeness of registers or lists used in differing data tracking systems and to estimate the number of disease events such as HIV, tuberculosis, dengue, influenza, and malaria diagnoses $\mathrm{s}^{20,21}$; mortality ${ }^{22-24}$; population sizes in hardto-reach groups ${ }^{25-28}$; and completeness of disease surveillance systems. ${ }^{22-24,28}$

Recently, satellite images have also been used to estimate population size, ${ }^{25,28}$ enumerate sample frames, ${ }^{29-34}$ target interventions such as indoor residual spraying, ${ }^{35,36}$ and monitor polio vaccination activities. ${ }^{37}$ In most MDA contexts, however, household locations are not recorded with GPSenabled devices; however, where they do exist, the completeness of household visitation by campaign workers can be 
compared against an enumeration of structures in the target area from satellite images.

This article provides the results of an effort to calculate epidemiologic coverage and program reach (household coverage) of four MDA and fMDA rounds using capture-recapture methods. The capture-recapture coverage estimates are also compared with the more commonly used TCS method for estimating MDA coverage. Satellite enumeration of households in the MDA trial area was used to assess populations that were missed by both the capture-recapture and TCS methods.

\section{METHODS}

Data sources. A full description of the trial has been published elsewhere. ${ }^{1,7}$ In summary, the trial assessed the impact of four rounds of community-wide MDA, where all household members were given dihydroartemisinin-piperaquine (DHAp) regardless of RDT results, versus fMDA, where DHAp was given to all eligible household members if anyone in the house had a positive RDT, on malaria parasite infection prevalence from November 2014 to March 2016. Data from each MDA and fMDA round were used to determine the number of households visited, total number of individuals, and testing and treatment status. Furthermore, two household surveys were conducted from April through May in 2015 and 2016 to establish the follow-up and final parasite infection prevalence in the study arms. ${ }^{7}$ Each survey was implemented approximately 2-3 months after the second and fourth MDA rounds, respectively, and included a TCS module with questions assessing MDA household visitation, testing, treatment, and reasons for not being visited, tested, or treated in the two preceding mass treatment rounds. All study data were collected on Android mobile phones by pairs of community health workers (CHWs) assigned to health catchment areas. A full description of treatment adherence has been published elsewhere. $^{38}$

An independent satellite enumeration exercise using Bing Map Imagery (Microsoft, Redmond, WA) was conducted in March 2015 to identify areas completely missed by the baseline household census, household surveys, and MDA rounds. The enumeration exercise consisted of manually pinpointing and classifying all structures based on size and assumed use. A total of 121,309 structures were enumerated in the 40 intervention catchments, of which 51,599 corresponded to GPS points provided by the MDA campaign for rounds 1 and 2 . The enumeration team consolidated this file to 50,364 potential household structures in the 40 catchments, where a central point in a cluster of structures was considered a household. This methodology has been described previously. ${ }^{35,36}$

Data analysis: program data. Table 1 summarizes the coverage definitions and source data. WHO coverage definitions were adapted to serve as a framework to organize the analysis of coverage and to further standardize terminology. ${ }^{39}$ Program coverage estimates were calculated by aggregating for each round and trial arm the number of individuals provided treatment divided by the total individuals listed by the field teams during the mass treatment campaign rounds.

Data analysis: capture-recapture data. Capture-recapture methods were used to estimate the total number of households in each catchment that should have been visited by

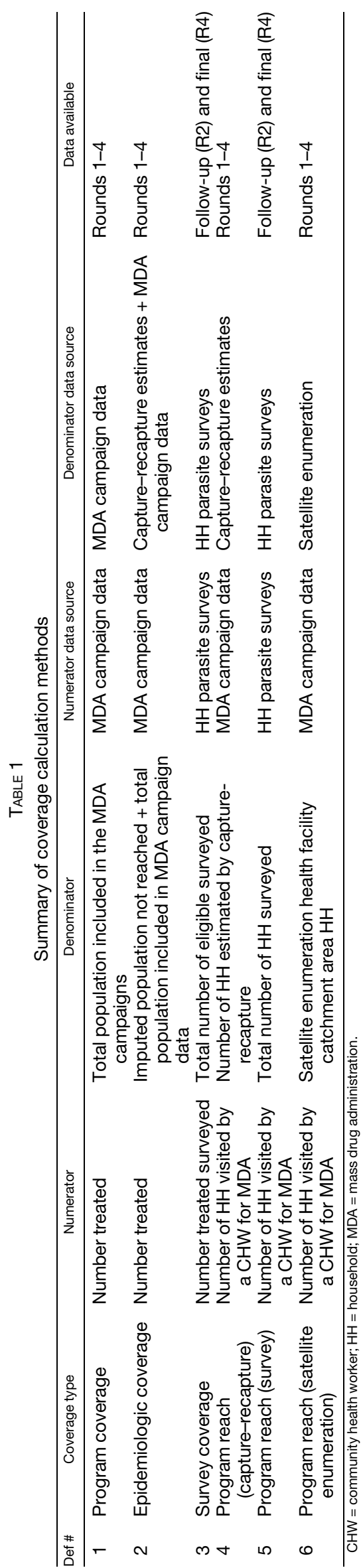


campaign field teams. Individuals from two independent lists - the campaign round datasets and the 2015 midpoint parasite survey-were matched using record linkage theory on the basis of name, age, gender, catchment, and household GPS coordinates. ${ }^{40}$ Figure 1 illustrates the timing of matched study activities. A household was considered matched from each campaign round to the survey if at least one enumerated household resident was matched. Matches were restricted to the same catchment area. The number of households per catchment was calculated using the Schnabel estimation methodology. ${ }^{41}$ There are three primary assumptions that must be met for this method: 1) the population is closed without gains or losses between mark-recapture, 2) sampling is random, and 3) all individuals have an equal chance of capture. ${ }^{20}$

Program reach was calculated by dividing the total reported number of households visited by the trial arm by the total number of households that should have been visited during campaign rounds based on capture-recapture. The total population not reached was imputed by multiplying the average household size for the trial area by the difference in the total number of households calculated by capture-recapture and the actual number of households visited. The denominator for epidemiologic coverage comprises the total enumerated population by round and arm and the imputed population not reached. Record matching and Schnabel analyses were performed in $R$ version 3.3.1 ( $R$ Foundation for Statistical Computing, Vienna, Austria) and manual verification in Microsoft Excel version 15.30. ${ }^{42}$ For Schnabel estimation, the Simple Fisheries Stock Assessment Methods package was used to corroborate manual estimation. ${ }^{43}$

Data analysis: satellite enumeration data. Each MDA round dataset was matched to the spatially enumerated household structure file based on geo-location, excluding houses with missing GPS points. All households from each campaign round were plotted, and a 40-m buffer was drawn around the household point. If plotted satellite structures fell within a buffered household, then the satellite structure was considered matched; after each round, the balance of structures not visited was matched to the next round until all rounds were plotted and matched. The structures not matched after comparison to round four of the trial were considered not visited during any campaign round. Program reach based on satellite enumeration was calculated by dividing the total number of structures visited by campaign teams by the total structures enumerated.

Data analysis: household survey data. Survey coverage is the percentage of survey respondents who reported being treated in rounds two and four divided by all residents recorded in the surveyed households. The percentage of surveyed households reporting being visited for rounds 2 and 4 comprises the program reach for each round by arm. Univariate and multivariable logistic regression analyses were performed to measure whether there were differences in demographics and household characteristics among survey respondents who received an MDA visit and those who did not. Calculations were performed using Stata 13.1 (StataCorp, College Station, TX) and adjusted for the cluster survey design. Coverages were aggregated by fMDA, MDA, and both arms.

\section{RESULTS}

Program data. The number of individuals and households enumerated during each campaign round decreased in rounds two and four, which occurred during the rainy season, compared with rounds 1 and 3 , which occurred during the dry season (Table 2). The average number of households visited for MDA and fMDA during rounds 1 and 3 was 17,883 and 17,854 , respectively, and decreased to 14,921 and 15,330 during rounds 2 and 4 , respectively. Program coverage ranged from $79.9 \%$ to $87.0 \%$ for the MDA trial arm and $10.2 \%$ to $26.6 \%$ for the fMDA trial arm across the four rounds. The low coverage and decrease in IMDA accurately reflects application of the trial protocol, where only people in households where someone was RDT positive received treatment, amid declining parasite prevalence over the trial period. ${ }^{7}$ Adherence information was collected for 181,534 of 336,821 DHAp $(53.9 \%)$ treatments administered during four rounds of MDA fMDA, of which 153,197 (84.4\%) reported completing the full course of DHAp. ${ }^{38}$ Approximately 340 mobile phones (three to 20 per catchment) were used during each MDA round for data collection.

Capture-recapture. The percentages of individuals from the midpoint household survey ( $n=8,142$ in 1,649 households) that were matched to individuals enumerated in campaign rounds 1 through 4 were $43.5 \%, 49.6 \%, 37.6 \%$, and $42.1 \%$, respectively. The resulting percentages of households from the survey where at least one matched individual from each campaign round was found were $70.4 \%, 78.6 \%, 66.3 \%$, and $70.8 \%$, respectively, by round. For a sensitivity analysis, considering the 9- to 10-month lag between the midpoint household survey and round 4 , the final household survey that occurred 2-3 months after round 4 was matched with the same methods. Results were comparable with $47.1 \%$ of individuals and $76.8 \%$ of households matched.

The total number of households calculated in the 40 catchment areas was 46,407 (95\% Cl: 44,345-48,671) (Table 3). The

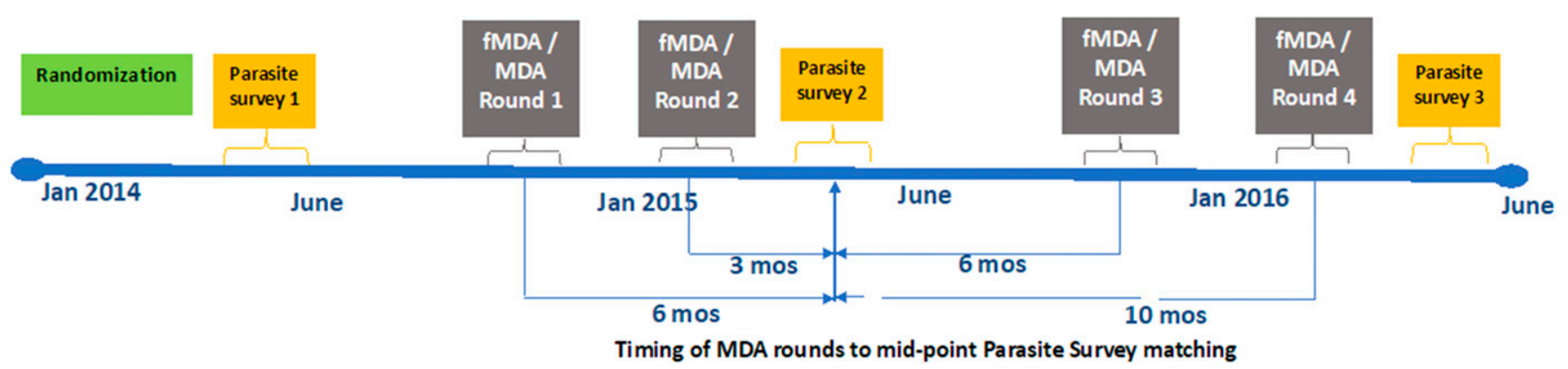

FIGURE 1. Study timeline and matching of rounds to parasite survey. This figure appears in color at www.ajtmh.org. 


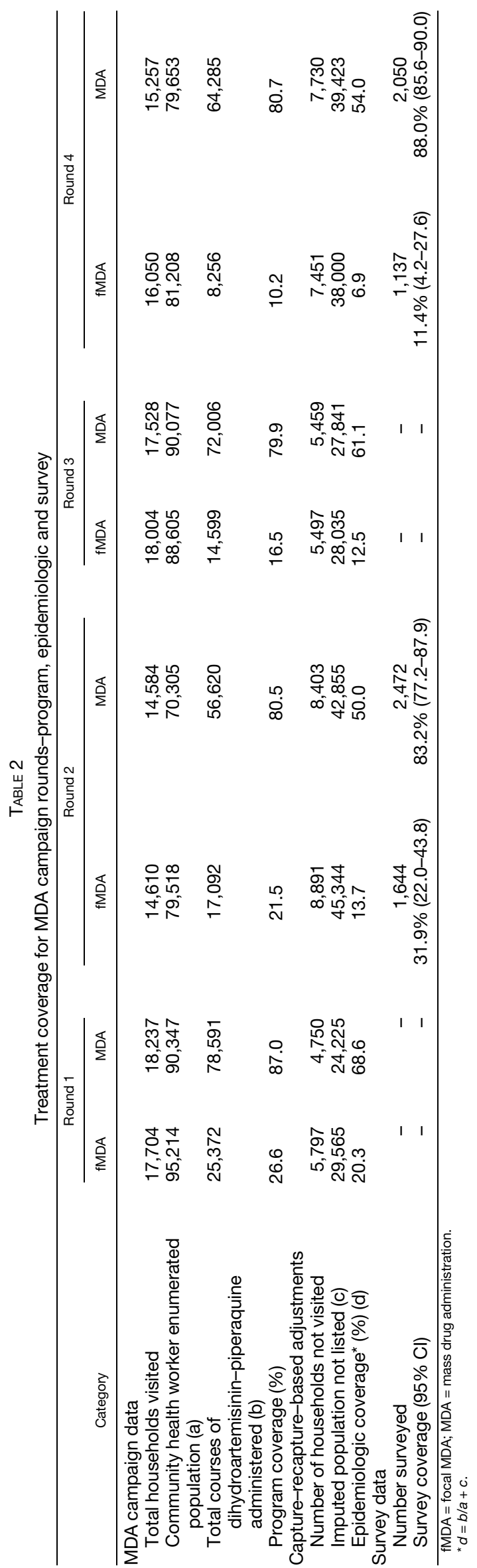

maximum number of reported households visited for all catchments in any round by $\mathrm{CHWs}$ was 39,482 . The total number of households from capture-recapture was $17.5 \%$ greater than the maximum number of households visited in any round and $5.2 \%$ less than the Ministry of Health administrative population estimates $(n=48,938)$.

Table 3 summarizes the program reach (i.e., household coverage) by trial arm and round using the capture-recapture method. The percentage of households reached in MDA ranged from a low of $63.4 \%(95 \% \mathrm{Cl}: 59.3-67.6)$ in round 2 to a high of $79.3 \%$ (95\% Cl: $74.2-84.5)$ in round 1 . For fMDA, results were similar with a low in round 2 of $62.2 \%(95 \% \mathrm{Cl}$ : $58.0-66.3)$ to a high of $76.6 \%(95 \% \mathrm{Cl}: 71.5-81.7)$ in round 3. Overall, if the maximum number of visited households across all campaign rounds was used as the numerator, then the program reach for at least one visit would have been $86.4 \%$ (95\% Cl: 80.8-92.0) in MDA and 83.5\% (95\% Cl: 78.0-89.1) in fMDA trial arms. Figures 2 and 3 illustrate variation in the household estimates from capture-recapture, campaign workers, and national estimates at the catchment level. Review of the study area and matching results demonstrated pockets of catchments that were never visited by campaign teams; principally in Namaila, Mapatizya, Luyaba, Mtendere, and Nanduba catchments.

Based on capture-recaptures results, the reported program coverage was adjusted by the imputed number of individuals in the estimated number of households not visited (Table 2) to calculate epidemiologic coverage. For MDA, the epidemiologic coverage ranged from $50.0 \%$ to $68.6 \%$. Declines were similar in rounds 2 and 4 (done during rainy seasons) as compared with rounds one and three (dry seasons) based on difficult logistics during the rainy seasons, as discussed earlier.

Satellite enumeration. A total of 50,634 probable household structures were enumerated, of which the percentage of structures visited in any round of MDA and fMDA by campaign teams was $68.4 \%$ (95\% Cl: $62.5-74.3)$ and $64.8 \%(95 \% \mathrm{Cl}$ : $58.1-71.5)$, respectively. This compares to an average of $71.1 \%$ from capture-recapture that were visited across all four rounds. The remaining structures not matched $(16,303)$ were not verified as being occupied or valid dwellings. GPS points were missing for $15.6 \%$ of households in rounds 1 and 2 and $6.9 \%$ in rounds 3 and 4 .

Survey data. In the MDA trial arm, the percentage of survey households being visited during rounds 2 and 4 was $72.2 \%$ $(n=759,95 \% \mathrm{Cl}: 62.4-80.2)$ and $83.4 \%(n=869,95 \% \mathrm{Cl}:$ 74.6-89.6), respectively. In the fMDA arm, the percentage of survey households being visited during rounds 2 and 4 was 53.9\% ( $n=729,95 \% \mathrm{Cl}: 44.6-63.0)$ and $66.2 \%(n=837,95 \%$ $\mathrm{Cl}$ : 52.0-78.0), respectively. Each survey estimate of program reach was greater than the program reach estimates based on capture-recapture and satellite enumeration besides round 2 of fMDA (Table 4). Table 1 presents comparison of program and survey coverages.

\section{DISCUSSION}

This study used multiple methods to calculate and triangulate the coverage of MDA and FMA interventions across four treatment rounds in a community-randomized controlled trial. The trial provided 336,821 doses of DHAp to 674,927 individuals, as per protocol, over four rounds, representing a massive effort to reduce the parasite reservoir. Results from 


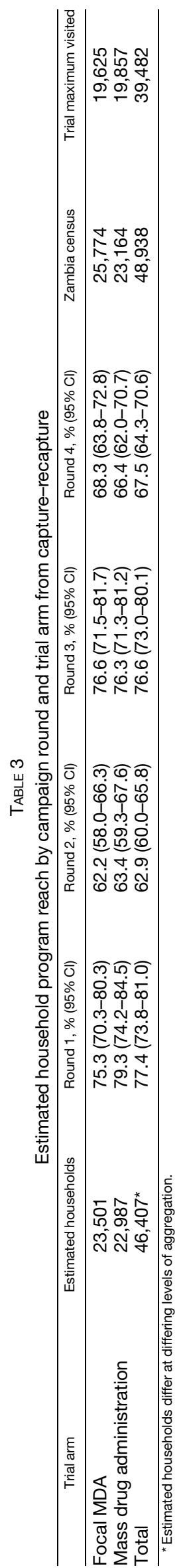

the household surveys examining treatment, visitation, testing, and RDT positivity after two of the four rounds suggest campaign staff program data were accurate and reliable, with differences being minor. However, relying exclusively on the program data for calculating coverage of DHAp and program reach would unknowingly lead to biased results. These methods do not inform who was potentially not visited and not offered treatment. The program data from this trial unequivocally established that many individuals were not treated during rounds 2 and 4 , which were during the rainy season. Trial implementers noted the decrease in the number of households visited after round 2 and commissioned satellite enumeration to better understand the number of potential households and the totality of unvisited areas. Capturerecapture was used after the trial to estimate the total number of households residing in the trial area that were potentially not visited and provided DHAp and used to estimate epidemiologic coverage.

Capture-recapture and satellite enumeration estimates of program reach were comparable to each other but lower than those relying solely on campaign data from $\mathrm{CHWs}$. Based on a fixed household denominator for each trial arm from capture-recapture, the trial program reach was less than household survey estimates for rounds 2 and 4 for MDA and round 4 for fMDA, whereas it was greater for round 2 for fMDA, within the bounds of the $\mathrm{Cl}$ for capture-recapture. When examined together, capture-recapture and satellite enumeration methodologies provided two estimates of the total number of households within which the 2010 official adjusted Zambian census estimate was the midpoint. When comparing each with the maximum number of households visited by campaign staff during any round in each catchment, these findings affirm that many households were either not visited or unavailable for testing and treatment during the campaign rounds. If plotted and overlapped with previous catchment boundaries from other work in the region, select areas of catchments that were not visited may have been misunderstood as being outside the trial implementation boundaries and erroneously excluded by campaign staff. ${ }^{44}$ Attempts to confirm structures as unoccupied in later rounds were inconsistent, and structures could not be reliably removed from the datasets before analysis. Although similar work in Zambia and Malawi has demonstrated the method's accuracy to correctly predict sleeping structures, without conclusive evidence that the structures enumerated herein had occupants at the time of the round visits, it is difficult to unequivocally affirm the precision of the capture-recapture population. ${ }^{31,34,45}$ Therefore, these coverage estimates are conservative, and actual program reach and epidemiologic coverage is likely greater.

These results illustrate the challenges community-based MDA programs face when calculating program reach and determining how to report epidemiologic coverage. This raises questions as to what the appropriate denominator should be to determine program reach and epidemiological coverage over a lengthy implementation period. Recent studies examining the variation in subnational populations highlight the importance of examining this issue further; relying on static denominators may overestimate the incidence of malaria by $30 \%$ because of substantial population movement. ${ }^{46}$ Other studies in this geographic area have also noted variation in intervention coverage by rounds and that subareas are visited consistently or not at all from round to round. ${ }^{5}$ Additional work 


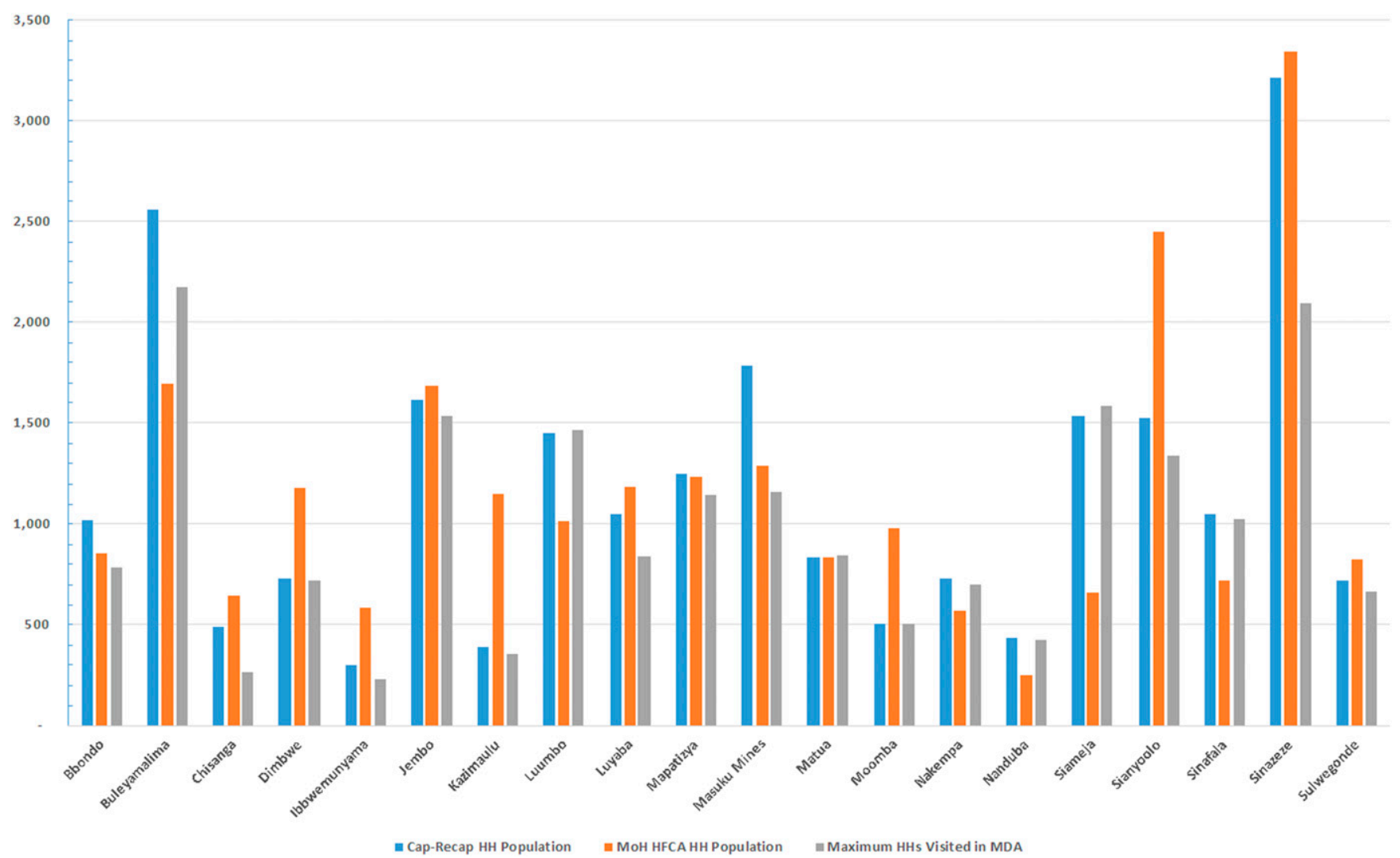

FIGURE 2. Comparison of capture-recapture, Ministry of Health administrative and maximum campaign round households per catchment for the mass drug administration trial arm. This figure appears in color at www.ajtmh.org.

has highlighted the inaccuracies of denominator data and the need for refined satellite enumeration and ground confirmation that accounts for migration and seasonal variation. ${ }^{47,48}$

In areas with dynamic population denominators, the challenge of providing complete coverage to all eligible individuals requires innovative strategies and improved MDA practices. Exploration of seasonal shifts of residents due to farming and nontraditional settings such as fish camps and boarding schools is needed to better inform program planning and to ensure that groups of individuals are not migrating to areas not covered by mass treatment and consequently at risk of serving as parasite reservoirs to areas previously treated. Furthermore, understanding how many potentially occupied structures are in a geographic target area and ensuring that they are visited each time to confirm that no one is residing there are paramount for consistent and reliable reporting. Adopting this as the best practice would address concerns over different denominators by rounds (which occurred during this trial) and better inform the appropriate MDA program reach.

Mass drug administration programs should consider additional studies of the feasibility of capture-recapture methods to ascertain its reliability and usefulness at estimating population. Although it is feasible to link structures over time through record linkage or spatial matching, there are limitations from data quality and degree of missing data, and these methods would not be available to the vast majority of MDA programs that do not use GPS-enabled devices and are administered by local health facility teams with paper registers. To this extent, satellite enumeration is a worthwhile expenditure if performed in advance of MDA activities and accompanied by micro-planning for campaign workers and ground-verification exercises. ${ }^{37}$ Recent work in Northern Nigeria for monitoring geographic coverage of polio campaign activities demonstrates the feasibility of alternate monitoring strategies and the benefits of the extensive use of mapped settlements with population estimates and GPS tracking of workers. $^{37,49}$ Furthermore, these methods have been extended to new contexts, given the lack of consensus mapping of catchment target areas. ${ }^{50}$

Limitations. This work primarily focused on examining the extent to which households in the study area may not have been visited by campaign staff. These results must consider several limitations. First, with respect to capture-recapture methods used herein, in this study, the household survey population that served as the "mark" was randomly selected from an enumeration list that predated the first campaign round. Data collectors for the campaign treatment rounds were not the same individuals as the survey workers and campaign teams worked independently in their catchments and were to provide blanket coverage to all resident households. Thus, we considered assumptions of randomness and equal selection met. With respect to a closed population, as assumed in other population-level uses of closed capturerecapture models, given the short time frame in which the data capture events occurred, we considered this met. ${ }^{21,23} \mathrm{Al}-$ though the data demonstrate that the population did change from round to round, the record linkage process was designed to find individuals from the survey houses, which were confirmed as present during April and May midway through the trial, and flag the household as having been visited. 


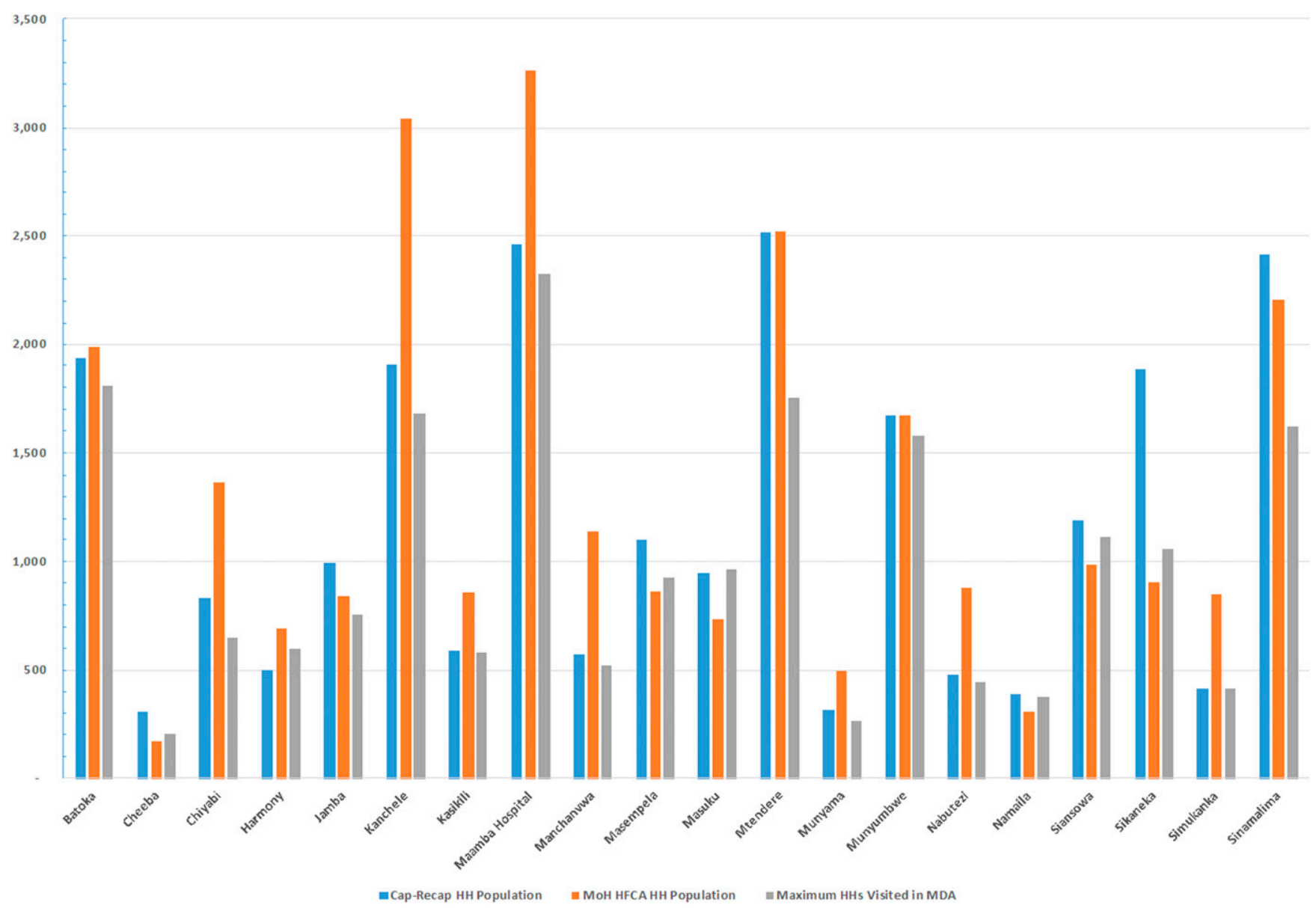

FIGURE 3. Comparison of capture-recapture, Ministry of Health administrative and maximum campaign round households per catchment for the focal MDA trial arm. This figure appears in color at www.ajtmh.org.

There are several limitations to relying on the TCS as a reference comparison. First, survey data were derived from responses by the head of the household, or adult representative, on behalf of all individuals in the house approximately 23 months after the drug distribution depending on the catchment. Proxy status was not recorded for testing and treatment data; thus, the accuracy of these responses may be affected by recall and response biases, particularly in fMDA where RDT positivity was low and estimates of program reach were consistently lower than in MDA. Although the recent literature suggests recall may be accurate up to 6-12 months after
MDA, ${ }^{51,52}$ the TCS module was bundled within a large, complex survey that took 1-2 hours to administer, which is not ideal for assessing coverage alone. Notwithstanding these limitations, this methodology was the most robust post-distribution coverage survey design in this trial and affirmed DHAp provision, RDT testing, and RDT positivity for the trial arms.

During large-scale trials or programs relying on electronic data capture, loss of information from hardware failure is possible and data entry quality may vary markedly by enumerator. In this trial, approximately 340 phones were used during each MDA round for household visits. Familiarity and

TABLE 4

Comparison of program reach estimates for MDA and fMDA rounds 2 and 4 from survey, capture-recapture, and satellite enumeration

\begin{tabular}{|c|c|c|c|c|}
\hline & MDA round 2 & MDA round 4 & fMDA round 2 & fMDA round 4 \\
\hline Category & Prop $(95 \% \mathrm{Cl})$ & Prop $(95 \% \mathrm{Cl})$ & Prop $(95 \% \mathrm{Cl})$ & Prop $(95 \% \mathrm{Cl})$ \\
\hline Household surveys, $n$ & 755 & 896 & 725 & 851 \\
\hline Any visit by a CHW for MDA & $0.89(0.84-0.93)$ & $0.90(0.84-0.95)$ & $0.63(0.54-0.72)$ & $0.74(0.61-0.88)$ \\
\hline Of $\mathrm{HH}$ visited, reporting two $\mathrm{HH}$ visits & $0.76(0.66-0.84)$ & $0.45(0.27-0.63)$ & $0.75(0.67-0.83)$ & $0.55(0.41-0.69)$ \\
\hline Program reach* ${ }^{*}$ & $0.72(0.62-0.80)$ & $0.83(0.76-0.91)$ & $0.54(0.45-0.63)$ & $0.66(0.53-0.79)$ \\
\hline Capture-recapture, $n$ & 23,197 & 23,197 & 23,413 & 23,413 \\
\hline Program reach & $0.63(0.45-0.81)$ & $0.66(0.47-0.85)$ & $0.62(0.44-0.81)$ & $0.69(0.48-0.89)$ \\
\hline Satellite enumeration, $n$ & 24,574 & 24,574 & 24,960 & 24,960 \\
\hline $\begin{array}{l}\text { Program reach by a } \mathrm{CHW} \text { at any time } \\
\text { during trial }\end{array}$ & \multicolumn{2}{|c|}{$0.65(0.58-0.71)$} & \multicolumn{2}{|c|}{$0.68(0.62-0.74)$} \\
\hline
\end{tabular}

${ }^{\star}$ Coverage data were not collected for rounds $1+3$ 
improvements to the software led to decreased information loss for treatment data and geo-location information for structures visited in later rounds but ranged from $6.9 \%$ to $15.4 \%$ by round. Thus, the actual treatment coverage may be greater than what the programmatic records indicate in certain catchments. For a sensitivity analysis of structural matching, a 50 -meter buffer increased matching from $67.6 \%$ to $71.0 \%$ and for a 75 -meter buffer to $76.1 \%$. These issues may also have affected the success of satellite enumeration matching and capture-recapture estimation biasing results downward.

\section{CONCLUSION}

As MDA for malaria moves from trial conditions to routine administration under certain programmatic and transmission circumstances, malaria MDA programs must consider methods for reporting and validating treatment coverage and program reach similar to other intervention programs. Reaching individuals in a campaign is only one part of a process that must also include adherence to the treatment course for ensuring that the effective drug coverage is as high as possible. Known issues in relying on program coverage across numerous MDA programs from decades of MDA experience must be taken into account before launching large-scale malaria MDA activities. The NMCC manages a robust, multifaceted malaria control program, but this study highlights challenges encountered even under clinical trial conditions. With reticence toward using MDA as an elimination strategy well founded in the documented experience of its failure and its contribution to drug resistance, malaria MDA programs should consider the utility of satellite enumeration for planning MDA campaigns, particularly in areas where it has not occurred before, to aid in the segmentation of administrative areas for implementation and assessing the number of potential households.

Received September 6, 2019. Accepted for publication April 8, 2020.

Published online July 2, 2020.

Acknowledgments: We express our gratitude to the study respondents in Southern Province for participating in this study. We also express our gratitude to the Zambia Ministry of Health at all levels. At the Provincial Health Office, we are especially grateful for the support of Jelita Chinyonga, provincial medical officer, and her staff for moving this effort forward. We would like to thank Muleba Matafwali, Kedrick Katonga, Sosenna Assefa, Juliana Ngalande, Hazel Chabala, and Elisabeth Wilhem for their enduring support during study implementation. We also thank the Bill \& Melinda Gates Foundation for their financial support.

Financial support: The trial was an investigator-initiated study supported by a grant from the Bill \& Melinda Gates Foundation.

Disclaimer: The funding source had no role in the conduct, analysis, or interpretation of results of the study. All authors had full access to all the data in the study.

Authors' addresses: Timothy P. Finn, Joshua O. Yukich, Travis R. Porter, Joseph Keating, and Thomas P. Eisele, Department of Tropical Medicine, Center for Applied Malaria Research and Evaluation, Tulane University School of Public Health and Tropical Medicine, New Orleans, LA, E-mails: tfinn2@tulane.edu, jyukich@tulane.edu, tporter1@tulane.edu, jkeating@tulane.edu, and teisele@tulane.edu. Adam Bennett, Malaria Elimination Initiative, Global Health Group, University of California San Francisco, San Francisco, CA, E-mail: adam.bennett@ucsf.edu. Christopher Lungu, Kafula Silumbe, and John M. Miller, PATH Malaria Control and Elimination Partnership in Africa (MACEPA), Lusaka, Zambia, E-mails: clungu@path.org, ksilumbe@path.org, and jmiller@path.org. Busiku Hamainza and Elizabeth Chizema Kawesha, National Malaria Elimination Centre, Zambia Ministry of Health, Chainama Hospital, Lusaka, Zambia, E-mails: bossbusk@gmail.com and e.chizema5@gmail.com. Ruben O. Conner and Richard W. Steketee, PATH Malaria Control and Elimination Partnership in Africa, Seattle, WA, E-mails: rconner@ path.org and rsteketee@path.org.

This is an open-access article distributed under the terms of the Creative Commons Attribution (CC-BY) License, which permits unrestricted use, distribution, and reproduction in any medium, provided the original author and source are credited.

\section{REFERENCES}

1. Eisele TP et al., 2016. Short-term impact of mass drug administration with dihydroartemisinin plus piperaquine on malaria in Southern Province Zambia: a cluster-randomized controlled trial. J Infect Dis 214: 1831-1839.

2. Brady OJ et al., 2017. Role of mass drug administration in elimination of Plasmodium falciparum malaria: a consensus modelling study. Lancet Glob Health 5: e680-e687.

3. Stuckey EM, Miller JM, Littrell M, Chitnis N, Steketee R, 2016. Operational strategies of anti-malarial drug campaigns for malaria elimination in Zambia's Southern Province: a simulation study. Malar J 15: 1-14.

4. Gerardin J, Bever CA, Hamainza B, Miller JM, EckhoffPA, Wenger EA, 2016. Optimal population-level infection detection strategies for malaria control and elimination in a spatial model of malaria transmission. PLoS Comput Biol 12: e1004707.

5. Nikolov M, Bever CA, Upfill-Brown A, Hamainza B, Miller JM, Eckhoff PA, Wenger EA, Gerardin J, 2016. Malaria elimination campaigns in the Lake Kariba region of Zambia: a spatial dynamical model. PLoS Comput Biol 12: e1005192.

6. Newby $G$ et al., 2015. Review of mass drug administration for malaria and its operational challenges. Am J Trop Med Hyg 93: 125-134.

7. Eisele TP et al., 2015. Assessing the effectiveness of householdlevel focal mass drug administration and community-wide mass drug administration for reducing malaria parasite infection prevalence and incidence in Southern Province, Zambia: study protocol for a community randomized controlled trial. Trials 16: 347.

8. Astale T et al., 2018. Population-based coverage survey results following the mass drug administration of azithromycin for the treatment of trachoma in Amhara, Ethiopia. PLoS Negl Trop Dis 12: e0006270-13.

9. Griswold E et al., 2018. Evaluation of treatment coverage and enhanced mass drug administration for onchocerciasis and lymphatic filariasis in five local government areas treating twice per year in Edo state, Nigeria. Am J Trop Med Hyg 99: 396-403.

10. Baker MC, Krotki K, Sankara DP, Trofimovich L, Zoerhoff KL, Courtney L, Chowdhury D, Linehan M, 2013. Measuring treatment coverage for neglected tropical disease control programs: analysis of a survey design. Am J Epidemiol 178: 268-275.

11. Cromwell EA, Ngondi J, McFarland D, King JD, Emerson PM, 2012. Methods for estimating population coverage of mass distribution programmes: a review of practices in relation to trachoma control. Trans $R$ Soc Trop Med Hyg 106: 588-595.

12. Shuford K, Were F, Awino N, Samuels A, Ouma P, Kariuki S, Desai $\mathrm{M}$, Allen DR, 2016. Community perceptions of mass screening and treatment for malaria in Siaya County, western Kenya. Malaria Journal 15: 71.

13. Adhikari RK, Sherchand JB, Mishra SR, Ranabhat K, Wagle RR, 2014. Awareness and coverage of mass drug administration for elimination of lymphatic filariasis: a community based cross sectional study in Nepal. J Community Health 40: 34-40.

14. Poirot E, Skarbinski J, Sinclair D, Kachur SP, Slutsker L, Hwang J, 2013. Mass drug administration for malaria. Cochrane Database Syst Rev 12: CD008846. Available at: http://onlinelibrary. wiley.com/doi/10.1002/14651858.CD008846.pub2/pdf/ standard.

15. Seidlein von L, Greenwood BM, 2003. Mass administrations of antimalarial drugs. Trends Parasitol 19: 452-460. 
16. Cromwell EA, Ngondi J, Gatpan G, Becknell S, Kur L, McFarland D, King JD, Emerson PM, 2009. Estimation of population coverage for antibiotic distribution for trachoma control: a comparison of methods. Int Health 1: 182-189.

17. Mathieu E, Deming M, Lammie PJ, McLaughlin SI, Beach MJ, Deodat DJ, Addiss DG, 2003. Comparison of methods for estimating drug coverage for filariasis elimination, Leogane Commune, Haiti. Trans R Soc Trop Med Hyg 97: 501-505.

18. Woodhall DM, Mkwanda S, Dembele M, Lwanga H, Drexler N, Dubray C, Harris J, Worrell C, Mathieu E, 2014. Exploring innovative ways to conduct coverage surveys for neglected tropical diseases in Malawi, Mali, and Uganda.Acta Trop 132: 119-124.

19. Armstrup SC, McDonald TL, Manly BF, eds. 2005. Handbook of Capture-Recapture Analysis. Princeton, NJ: Princeton University Press.

20. Seber GAF, 1982. The Estimation of Animal Abundance and Related Parameters, 2nd edition. London, United Kingdom: C. Griffin \& Co., Ltd.

21. Chao A, Tsay PK, Lin SH, Shau WY, Chao DY, 2001. The applications of capture-recapture models to epidemiological data. Statist Med 20: 3123-3157.

22. Carter KL, Williams G, Tallo V, Sanvictores D, Madera H, Riley I, 2011. Capture-recapture analysis of all-cause mortality data in Bohol, Philippines. Popul Health Metr 9: 9.

23. Lewden $C$ et al.; 2000 Study Group, 2000. Number of deaths among HIV-infected adults in France in 2000, three-source capture-recapture estimation. Epidemiol Infect 134: 1345-1352.

24. Roberts B, Morgan OW, Sultani MG, Nyasulu P, Rwebangila S, Myatt M, Sondorp E, Chandramohan D, Checchi F, 2010. A new method to estimate mortality in crisis-affected and resourcepoor settings: validation study. Int J Epidemiol 39: 1584-1596.

25. Vong S, Goyet S, Ly S, Ngan C, Huy R, Duong V, Wichmann O, Letson GW, Margolis HS, Buchy P, 2012. Under-recognition and reporting of dengue in Cambodia: a capture-recapture analysis of the National Dengue Surveillance System. Epidemiol Infect 140: 491-499.

26. Peters TR, Snively BM, Suerken CK, Bischoff W, Vannoy L, Blakeney E, Bischoff T, Palavecino E, Sherertz R, Poehling KA, 2016. Estimating the burden of pandemic infectious disease: the case of the second wave of pandemic influenza H1N1 in Forsyth county, North Carolina. NC Med J 77: 15-22.

27. Héraud-Bousquet V, Lot $F$, Esvan $M$, Cazein F, Laurent $C$, Warszawski J, Gallay A, 2012. A three-source capturerecapture estimate of the number of new HIV diagnoses in children in France from 2003-2006 with multiple imputation of a variable of heterogeneous catchability. BMC Infect Dis 12: 251.

28. Eisele TP, Lindblade KA, Rosen DH, Odhiambo F, Vulule JM, Slutsker L, 2003. Evaluating the completeness of demographic surveillance of children less than five years old in western Kenya: a capture-recapture approach. Am J Trop Med Hyg 69: 92-97.

29. Wampler PJ, Rediske RR, Molla AR, 2013. Using ArcMap, google earth, and global positioning systems to select and locate random households in rural Haiti. Int $J$ Health Geogr 12: 1.

30. Chang AY, Parrales ME, Jimenez J, Sobieszczyk ME, Hammer SM, Copenhaver DJ, Kulkarni RP, 2009. Combining google earth and GIS mapping technologies in a dengue surveillance system for developing countries. Int J Health Geogr 8: 49.

31. Escamilla V, Emch M, Dandalo L, Miller WC, Martinson F, Hoffman I, 2014. Sampling at community level by using satellite imagery and geographical analysis. Bull World Health Organ 92: 690-694.

32. Haenssgen MJ, 2015. Satellite-aided survey sampling and implementation in low- and middle-income contexts: a lowcost/low-tech alternative. Emerg Themes Epidemiol 12: 20.

33. Pearson AL, Rzotkiewicz A, Zwickle A, 2015. Using remote, spatial techniquesto select a random household sample in a dispersed, semi-nomadic pastoral community: utility for a longitudinal health and demographic surveillance system. Int $J$ Health Geogr 14: 1-10. doi: 10.1186/s12942-015-0026-4.

34. Lowther SA, Curriero FC, Shields T, Ahmed S, Monze M, Moss WJ, 2009. Feasibility of satellite image-based sampling for a health survey among urban townships of Lusaka, Zambia. Trop Med Int Health 14: 70-78.

35. Kamanga A, Renn S, Pollard D, Bridges DJ, Chirwa B, Pinchoff J, Larsen DA, Winters AM, 2015. Open-source satellite enumeration. Malar J 14: 11-7. doi: 10.1186/s12936-015-0831-z.

36. Pinchoff $J$ et al., 2016. Targeting indoor residual spraying. Malar $J$ 15: 11-6. doi: 10.1186/s12936-015-1073-9.

37. Barau I, Zubairu M, Mwanza MN, Seaman VY, 2014. Improving polio vaccination coverage in Nigeria through the use of geographic information system technology. J Infect Dis 210 (Supp/ 1): S102-S110.

38. Finn TP et al., 2020. Adherence to mass drug administration of dihydroartemisinin-piperaquine and Plasmodium falciparum clearance in Southern Province, Zambia. Am J Trop Med Hyg 103 (Supp/ 2): 37-45.

39. World Health Organization, 2017. Coverage Evaluation Surveys for Preventive Chemotherapy, 1-54. Geneva, Switzerland: World Health Organization.

40. Blakely T, Salmond C, 2002. Probabilistic record linkage and a method to calculate the positive predictive value. Int J Epidemiol 31: 1246-1252.

41. Krebs CJ, 1998. Ecological Methodology, 2nd edition. Menlo Park, CA: Benjamin Cumings.

42. R Core Team, 2018. R: a Language and Environment for Statistical Computing. Vienna, Austria: R Foundation for Statistical Computing. Available at: https://www.R-project.org/.

43. Ogle DH, Wheeler P, Dinno A, 2020. FSA: Fisheries Stock Analysis. R package version 0.8.30.9000. Available at: https:// github.com/droglenc/FSA.

44. Larsen DA, Bennett A, Silumbe K, Hamainza B, Yukich JO, Keating JA, Littrell M, Miller JM, Steketee RW, Eisele TP, 2015. Population-wide malaria testing and treatment with rapid diagnostic tests and artemether-lumefantrine in Southern Zambia: a community randomized step-wedge control trial design. Am J Trop Med Hyg 92: 913-921.

45. Bridges DJ, Pollard D, Winters AM, Winters B, Sikaala C, Renn S, Larsen DA, 2018. Accuracy and impact of spatial aids based upon satellite enumeration to improve indoor residual spraying spatial coverage. Malar J 17: 1-8. doi: 10.1186/s12936-0182236-2.

46. Erbach-Schoenberg EZ et al., 2016. Dynamic denominators: the impact of seasonally varying population numbers on disease incidence estimates. Popul Health Metr 14: 1-10. doi: 10.1186/ s12963-016-0106-0.

47. Tatem AJ, 2014. Mapping the denominator: spatial demography in the measurement of progress. Int Health 6: 153-155.

48. Tatem AJ, 2014. Mapping population and pathogen movements. Int Health 6: 5-11.

49. Gali $\mathrm{E}$ et al., 2016. Revised household-based microplanning in polio supplemental immunization activities in Kano state, Nigeria. 2013-2014. J Infect Dis 213 (Supp/ 3): S73-S78.

50. Rosencrans LC, Sume GE, Kouontchou J-C, Voorman A, Anokwa Y, Fezeu M, Seaman VY, 2017. Mapping for health in Cameroon: polio legacy and beyond. J Infect Dis 216 (Suppl 1): S337-S342.

51. Budge PJ, Sognikin E, Akosa A, Mathieu EM, Deming M, 2016. Accuracy of coverage survey recall following an integrated mass drug administration for lymphatic filariasis, schistosomiasis, and soil-transmitted helminthiasis. PLoS Negl Trop Dis 10: e0004358.

52. Cromwell EA, King JD, McPherson S, Jip FN, Patterson AE, Mosher AW, Evans DS, Emerson PM, 2013. Monitoring of mass distribution interventions for trachoma in Plateau state, Nigeria. PLoS Negl Trop Dis 7: e1995. 